\title{
Tumor-Targeted, Cytoplasmic Delivery of Large, Polar Molecules using a pH-Low Insertion Peptide
}

\author{
Alexander A. Svoronos ${ }^{1,2}$, Raman Bahal ${ }^{3,4}$, Mohan C. Pereira ${ }^{1,5}$, Francisco N. Barrera ${ }^{1,6}$, \\ John C. Deacon ${ }^{1}$, Marcus Bosenberg ${ }^{7}$, Daniel DiMaio ${ }^{1,3,8}$, Peter M. Glazer ${ }^{3}$, Donald M. \\ Engelman ${ }^{1, *}$ \\ ${ }^{1}$ Department of Molecular Biophysics and Biochemistry, Yale University, New Haven, CT 06511, \\ USA \\ ${ }^{2}$ Department of Biomedical Engineering, Yale University, New Haven, CT 06511, USA \\ ${ }^{3}$ Department of Therapeutic Radiology, Yale University, New Haven, CT 06511, USA \\ ${ }^{4}$ Department of Pharmaceutical Sciences, University of Connecticut, Storrs, CT 06269, USA \\ ${ }^{5}$ Department of Science \& Mathematics, Cedarville University, Cedarville, OH 45314, USA \\ ${ }^{6}$ Department of Biochemistry \& Cellular and Molecular Biology, University of Tennessee, \\ Knoxville, TN 37996, USA \\ ${ }^{7}$ Department of Dermatology, Yale University, New Haven, CT 06511, USA \\ ${ }^{8}$ Department of Genetics, Yale University, New Haven, CT 06511, USA
}

\begin{abstract}
Tumor-targeted drug delivery systems offer not only the advantage of an enhanced therapeutic index, but also the possibility of overcoming the limitations that have largely restricted drug design to small, hydrophobic, "drug-like" molecules. Here, we explore the ability of a tumor-targeted delivery system centered on the use of $\mathrm{pH}$-low insertion peptide (pHLIP) to directly deliver moderately polar, multi-kDa molecules into tumor cells. A pHLIP is a short, $\mathrm{pH}$-responsive peptide capable of inserting across a cell membrane to form a transmembrane helix at acidic $\mathrm{pH}$. pHLIP targets the acidic tumor microenvironment with high specificity, and a drug attached to the inserting end of pHLIP can be translocated across the cell membrane during the insertion process.
\end{abstract}

\footnotetext{
"Corresponding Author: Mailing Address: 266 Whitney Ave., P.O. Box 208114, New Haven, CT 06520-8114; Phone: 203-432-5600; donald.engelman@yale.edu.

Author's Contributions

A.A.S., R.B., M.C.P., F.N.B., J.C.D, M.B., D.D., P.M.G., and D.M.E. designed the research. A.A.S., R.B., M.C.P., and F.N.B. developed the methodology. R.B. synthesized the PNAs. A.A.S. and M.C.P. acquired the data. A.A.S., M.C.P., J.C.D., and D.M.E. analyzed and interpreted the data. A.A.S., D.M.E., D.D., P.M.G., F.N.B., J.C.D., and M.B. wrote the manuscript.

Description of Supporting Information

Supporting Information. Chromatograms (SI Figure 1) and mass spectrometry results (SI Table 1) for pHLIP conjugates, control fluorescence emission and CD spectra (SI Figure 2), and confocal microscopy images of pHLIP delivery of PNA cargoes to cultured cells (SI Figure 3).

Disclosure of Potential Conflicts of Interest

M.B. is a consultant for Eli Lilly and Company. P.M.G. is a consultant to and has equity interests in Trucode Gene Repair, Inc. of San Francisco, CA and Cybrexa Therapeutics, Inc. of New Haven, CT and has an equity interest in Patrys, Ltd. of Melbourne, Australia. D.M.E. is a founder of and has equity interests in pHLIP, Inc., which is developing applications of the pHLIP technology. These companies did not fund any part of the work reported in this paper.
} 
We investigate the ability of pHLIP to deliver peptide nucleic acid (PNA) cargoes of varying sizes across lipid membranes. We find that pHLIP effectively delivers PNAs up to $\sim 7 \mathrm{kDa}$ into cells in a $\mathrm{pH}$-dependent manner. In addition, pHLIP retains its tumor targeting capabilities when linked to cargoes of this size, although the amount delivered is reduced for PNA cargoes greater than $\sim 6$ $\mathrm{kDa}$. As drug-like molecules are traditionally restricted to sizes of $\sim 500 \mathrm{Da}$, this constitutes an order-of-magnitude expansion in the size range of deliverable drug candidates.

\section{Graphical Abstract}

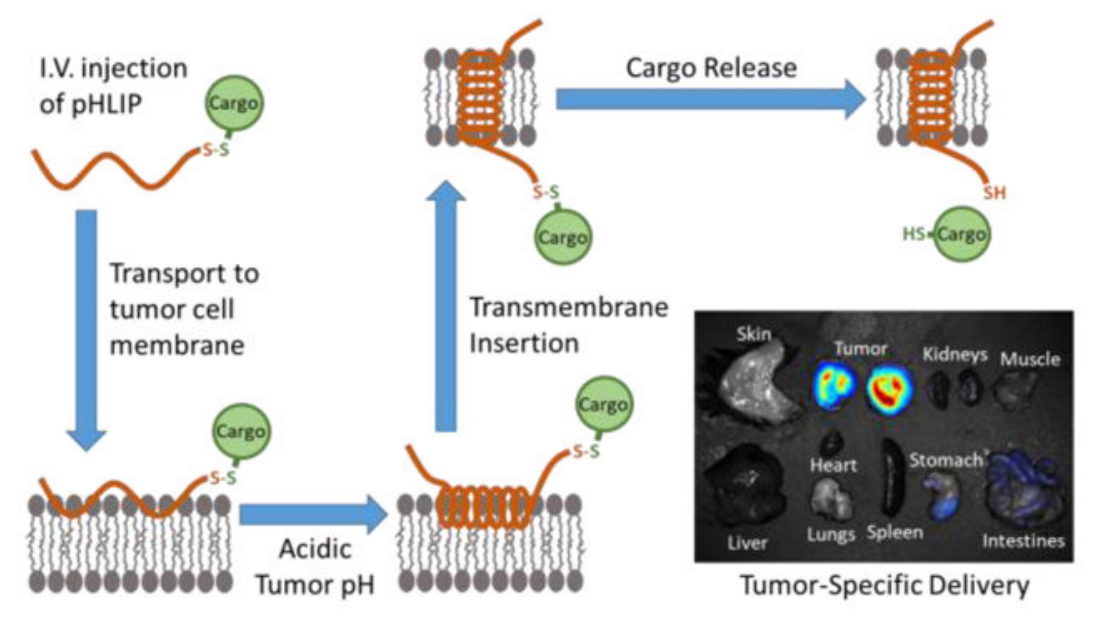

\section{Keywords}

pHLIP; tumor-targeted drug delivery; tumor acidity; transmembrane insertion; membrane permeability; drug size

\section{Introduction}

Achieving effective delivery of pharmaceuticals to intracellular sites of action is a contemporary challenge in drug development. While there are numerous obstacles to this endeavor, perhaps the most fundamental is the requirement for drugs to permeate the cell membrane in sufficient quantities so as to produce a therapeutic effect. As a result, the development of pharmaceuticals has largely been limited to either small, hydrophobic, "drug-like" molecules, which can passively diffuse through the cell membrane, or to biologics, which usually act through specific cell-surface receptors and, when intracellular delivery is necessary, undergo active transport through the membrane or uptake by endocytosis. ${ }^{1}$ The latter are obviously limited in terms of structure, mechanism of action, and the cell populations that can be targeted. On the other hand, small molecule drugs are confined to a limited range of physical properties, as charges, size, and hydrophobicity greatly influence passive diffusion through membranes. Indeed, small molecule drugs have traditionally been restricted to a molecular weight of less than $500 \mathrm{Da}$ and a $\operatorname{LogP}$ between 1 and $5 .^{2}$ While type 1 prodrugs can have active forms that are hydrophilic as a result of conversions inside a cell, the discovery of such compounds has been slow, and the prodrugs are still subject to the same size limitations as conventional small molecule drugs. ${ }^{3}$ Thus, 
there is a vast spectrum of potential therapeutic agents that remain unexplored in drug development efforts due to their non-drug-like physical properties.

Nevertheless, approaches exist that have the potential to deliver many of these agents. These approaches involve coupling the therapeutic agent to a molecule or complex of molecules that mediates cell entry. The most common of such mediators are nanoparticles. Therapeutic agents can be encapsulated into nanoparticles, which are then taken up by cells via endocytosis. As a result, drugs contained in nanoparticles can be much larger and more polar than passive diffusion-dependent drugs. Still, there are many challenges associated with nanoparticle delivery. First, their large size can make penetration through the blood vessel endothelium to the diseased tissue difficult. ${ }^{4}$ In addition, they are often too large to adequately diffuse through the interstitial fluid to reach all areas of the diseased tissues. ${ }^{4}$ After reaching the cells of interest, they must then adhere to the cell surface, often exploiting interactions with specific cell-surface receptors, before undergoing endocytosis. ${ }^{4}$ Finally, the contents of the nanoparticle must escape through two membranes, both that of the nanoparticle itself and that of the endosome, while avoiding degradation in the harsh endolysosomal environment. ${ }^{4}$ This can be especially challenging for polar cargoes. While some nanoparticle formulations show promise in overcoming these challenges, a more direct approach to cell penetration/delivery is often highly desirable.

Such an approach can be found in the use of $\mathrm{pH}$ (low) insertion peptides (pHLIPs). A pHLIP is a short, $\mathrm{pH}$-responsive peptide that inserts its $\mathrm{C}$-terminus across a cell membrane and forms a transmembrane helix when exposed to acidic conditions. ${ }^{5}$ A pHLIP can exist in three states: unstructured in solution (State 1), docked at a cell membrane but not inserted (State 2), and inserted across a cell membrane as a transmembrane alpha helix (State 3) (Figure 1). ${ }^{6}$ The State 2 to State 3 transition is the result of the protonation and consequent charge neutralization of aspartic acid residues in a pHLIP's membrane-spanning region at acidic $\mathrm{pH}^{5}$ This, in turn, increases the pHLIP's hydrophobicity and results in a change from an unstructured to a helical conformation, thereby making it energetically favorable for transmembrane insertion to occur. Importantly, therapeutic molecules can be attached as cargo to the inserting C-terminal end of pHLIP and thereby be directly inserted across the cell membrane at acidic $\mathrm{pH}$. Since the mechanism is insertion into the bilayer of the cell membrane, it is much more difficult to saturate the amount delivered than in the case of conventional biomarker targets.

The dependence of pHLIP insertion on acidic $\mathrm{pH}$ confers another significant advantage: pHLIP tends to accumulate in acidic diseased tissues with high specificity when administered in vivo, using cell surface acidity as a biomarker. Certain diseased tissues, most notably solid tumors, produce an acidic extracellular microenvironment as a consequence of their metabolism. ${ }^{7}$ The acidity is most pronounced at cell surfaces. ${ }^{8}$ As a result, pHLIP effectively targets tumors, and many studies have utilized pHLIP as the basis for tumortargeted imaging agents containing fluorophores or radioligands. ${ }^{9-15}$ Interestingly, pHLIP can also target other acidic diseased tissues, including sites of inflammation and ischemia. ${ }^{6}$ For the delivery of therapeutic agents, the targeting capability of pHLIP is especially appealing, since it has the potential to reduce toxicity from drug accumulation in nondiseased tissues. Indeed, pHLIP is currently being explored for the delivery of phalloidin ${ }^{16}$ 
and amanitin ${ }^{17}$, two powerful natural toxins, which, though not viable as drugs on their own due to toxicity, show promise as pHLIP-targeted therapeutics. Other potential therapeutic cargo molecules pHLIP has been used to deliver include paclitaxel ${ }^{18}$, antimicrobial peptides $^{19}$, monomethyl auristatins ${ }^{20,21}$, and peptide nucleic acids ${ }^{22-24}$. Usually, the therapeutic cargo molecule is attached to pHLIP via a disulfide bond, which is cleaved in the reducing environment of the targeted cell's cytosol, allowing the molecule to diffuse to its effector sites (Figure 1). The various cargoes that have previously been delivered by pHLIP, together with their respective molecular weights and $\log P / \log D$ values, are listed in Table 1. Notably, many of these cargoes are non-drug-like, with polar $\log P / \log D$ values and molecular weights greater than $500 \mathrm{Da}$.

Because of its properties, pHLIP has the potential to vastly expand the range of viable pharmaceutical compounds. pHLIP's free energies of cell membrane binding and insertion make it energetically favorable for many normally cell-impermeable molecules to translocate across the plasma membrane. ${ }^{24,25}$ It is thus useful to characterize the limits of pHLIP delivery in terms of the physical properties of the molecules it can deliver. Previously, hydrophobicity was examined using small (300-1000 Da) cyclic peptides, and it was found that pHLIP can translocate polar cargo molecules with cLogP values as low as -3 across cell membranes. ${ }^{26}$ However, the influence of cargo size on pHLIP-mediated delivery has not yet been systematically explored. Although the majority of cargoes previously delivered by pHLIP have been around $1 \mathrm{kDa}$ or less (Table 1), the successful delivery of peptide nucleic acids (PNAs) ${ }^{22-24}$ indicate that pHLIP is capable of delivering far larger molecules. PNAs are artificial RNA/DNA analogs that possess a polar, but chargeneutral peptide backbone instead of a negatively charged sugar phosphate backbone. ${ }^{27}$ It has been shown that pHLIP is capable of translocating PNAs into cells, ${ }^{22-24}$ whereas it cannot translocate similarly sized RNA molecules, presumably due to their highly polar backbones.

${ }^{24}$ Here, we use PNAs of varying length to explore the effects of cargo size on pHLIPmediated tumor targeting and delivery. We have chosen PNAs as our model cargo for four reasons: 1) Their size is easily tunable at constant $\log D$ by adjusting the number of nucleobases, 2) They are cell membrane-impermeable on their own (when not linked to a cationic cell-penetrating peptide sequence or to pHLIP), 3) They can be easily labeled with a fluorophore, thereby allowing tracking of their cell delivery and in vivo biodistribution, and 4) They have therapeutic potential as modulators of gene expression. ${ }^{28-31}$ Previously, pHLIP has been used to deliver PNAs that inhibit a microRNA ${ }^{22}$ and a long-noncoding RNA ${ }^{23}$, respectively. Although sufficient delivery for a therapeutic effect was achieved in both studies, it is important to note that tumor targeting by either pHLIP-PNA conjugate was not actually demonstrated in vivo. Here, we directly investigate the ability of pHLIP to target PNAs of different sizes to tumors in vivo while avoiding normal tissues. We also examine how PNA size affects the efficiency and kinetics of translocation by pHLIP across lipid membranes. In so doing, we find that pHLIP tumor targeting can remain highly specific for large (>6 kDa) molecules, and we establish guidelines for the size limitations of deliverable cargoes by pHLIP. 


\section{Experimental Methods}

\section{PNA Synthesis.}

PNAs were synthesized via solid phase synthesis using an MBHA (4-

Methylbenzhydrylamine) resin and Boc-monomers (A, T, C, G) purchased from ASM

Research Chemicals. 5-carboxytetramethylrhodamine (TAMRA) dye (free acid form)

(Biotium) and cysteine were linked to the $\mathrm{N}$-terminus and $\mathrm{C}$-terminus, respectively, of each

PNA via a Boc-miniPEG-3 linker (-ooo-). PNAs were further cleaved from the resin using a cleavage cocktail containing a 1:1:2:6 ratio of m-cresol: dimethyl sulfide:

trifluoromethanesulfonic acid (TFMSA): trifluoroacetic acid (TFA) followed by

precipitation using diethyl ether. The resulting PNAs were purified and characterized using reverse-phase high-performance liquid chromatography (HPLC) and matrix-assisted laser desorption/ionization-time of flight (MALDI-TOF) mass spectrometry, respectively. The concentration of PNAs was determined by UV-Vis spectroscopy using either the extinction coefficient of TAMRA $\left(90,000 \mathrm{M}^{-1} \mathrm{~cm}^{-1}\right.$ at $\left.560 \mathrm{~nm}\right)$ or an extinction coefficient equal to the sum of that of the individual PNA monomers at $260 \mathrm{~nm}\left(13,700 \mathrm{M}^{-1} \mathrm{~cm}^{-1}(\mathrm{~A}), 6,600 \mathrm{M}\right.$ ${ }^{-1} \mathrm{~cm}^{-1}(\mathrm{C}), 11,700 \mathrm{M}^{-1} \mathrm{~cm}^{-1}(\mathrm{G})$, and 8,600 $\mathrm{M}^{-1} \mathrm{~cm}^{-1}(\mathrm{~T})$ ), which agreed well with each other. The PNA sequences used in the study are listed in Table 1.

\section{Synthesis of pHLIP Constructs.}

The following pHLIP peptide sequence was ordered from CSBio (Menlo Park, CA, USA) with a 3-nitro-2-pyridinesulphenyl (NPys) group covalently masking the thiol of the cysteine that is the penultimate $\mathrm{C}$-terminal amino acid:

GGEQNPIYWARYADWLFTTPLLLLDLALLVDADEGTC(NPys)G. For PNA conjugation, pHLIP peptide was mixed with an equimolar concentration of PNA in argon-bubbled 3:1:1 DMSO:DMF:0.1 $\mathrm{mM} \mathrm{KH}_{2} \mathrm{PO}_{4}$ aqueous buffer, $\mathrm{pH} 4.5$. The solution was overlaid with argon and allowed to react on a shaker at room temperature for 3 days, protected from light. The NPys group on pHLIP prevented pHLIP dimer formation while acting as a leaving group to facilitate conjugation of the cysteine of pHLIP to the cysteine of the PNA via disulfide bond formation. Under these conditions, the approximate yield of the reaction was 30-40\%.

Non-cleavable pHLIP-TAMRA (without a PNA) was formed by conjugating the same sequence of pHLIP, but without an NPys group, to TAMRA-5-maleimide (Invitrogen) in DMSO at a molar ratio of 1.4:1 TAMRA:pHLIP. The reaction was incubated at room temperature for 6 hours, followed by $4^{\circ} \mathrm{C}$ for several days until the reaction reached completion, as determined by analytical HPLC.

Each resulting pHLIP construct was purified by reverse-phase HPLC with an Agilent Zorbax semi-prep $9.4 \times 250 \mathrm{~mm}$ SB-C18 column and a water/acetonitrile mobile phase gradient containing $0.1 \%$ trifluoroacetic acid. The pHLIP-PNAs would elute at $\sim 28$ minutes with the following settings: flow rate, $2 \mathrm{~mL} / \mathrm{min}$.; 5 minutes at 80:20 water:acetronitrile, followed by a linear gradient from 80:20 to 20:80 water:acetonitrile over 30 minutes, followed by 5 minutes at 20:80 water:acetonitrile, then return to 80:20 water:acetonitrile for 5 minutes. For pHLIP-PNAs, MALDI-TOF mass spectrometry was used for peak identification and molecular weight verification. For non-cleavable pHLIP-TAMRA, liquid 
chromatography-mass spectrometry was used. An HPLC chromatogram depicting the products of a typical pHLIP-PNA conjugation reaction, along with purity chromatograms for each pHLIP construct, is shown in SI Figure 1. The mass spectrometry results together with the corresponding expected masses for each construct are listed in SI Table 1. For use in experiments, TAMRA-labeled products were quantified by absorbance at $560 \mathrm{~nm}$ using an extinction coefficient of $90,000 \mathrm{M}^{-1} \mathrm{~cm}^{-1}$.

\section{Measurement of Octanol-Water Distribution Coefficient (LogD).}

Phosphate buffered saline (PBS), pH 7.4 was purchased from ThermoFisher and contained the following formulation: $155.17 \mathrm{mM} \mathrm{NaCl}, 1.06 \mathrm{mM} \mathrm{KH}_{2} \mathrm{PO}_{4}, 2.97 \mathrm{mM} \mathrm{Na}_{2} \mathrm{HPO}_{4}$. PBS, pH 6.2 and PBS-citrate, $\mathrm{pH} 4.0$ were created by the addition of concentrated $\mathrm{HCl}$ and 3.2 $\mathrm{mM}$ citrate, respectively. Solutions of 1-octanol saturated PBS or PBS-citrate were prepared by mixing on a shaker overnight and allowing the two phases to separate by standing for 2 hours. Buffer $\mathrm{pH}$ was adjusted using concentrated $\mathrm{HCl}$ or sodium hydroxide and the solution re-saturated with octanol by mixing until a stable $\mathrm{pH}$ at the desired value was achieved. For LogD measurements, $0.5-1 \mu \mathrm{M}$ of PNA and $100 \mu \mathrm{M}$ TCEP (to prevent PNA disulfide dimerization) were dissolved in $300 \mu \mathrm{L}$ of octanol-saturated PBS, and the absorbance was measured at $560 \mathrm{~nm}$ (for TAMRA). Afterward, $6 \mathrm{~mL}$ of PBS-saturated 1-octanol was added, and the resulting mixture was shaken vigorously and then incubated overnight on a rotoshaker. The following day, the mixture was phase-separated by centrifugation at $3000 \times \mathrm{g}$ for 5 minutes, and the absorbance of the aqueous phase was measured again at $560 \mathrm{~nm}$. The concentration of PNA in the octanol phase was inferred by subtraction of the absorbance in the aqueous phase after mixing with octanol from the absorbance before mixing and dividing by 20 , to account for the 20:1 octanol:PBS volume ratio. The LogD was calculated as the logarithm of the ratio of the concentration in the octanol phase to the concentration in the aqueous phase after octanol extraction.

\section{Preparation of POPC Vesicles.}

$50 \mathrm{~nm}$ POPC (Avanti Polar Lipids, Inc.) lipid vesicles were prepared by extrusion technique. First, POPC in chloroform was evaporated in a glass flask for 1 hour on a rotary evaporator at room temperature until a thin, even film on the sides of the flask was produced. Traces of organic solvent were subsequently removed by overnight evaporation under high vacuum. The resulting film was then hydrated in $\mathrm{pH} 8.0,10 \mathrm{mM}$ PBS for 30 minutes. The hydrated lipid suspension was subjected to 10 freeze/thaw cycles by alternately placing the flask in a liquid nitrogen bath and warm water bath. The mixture was then sonicated and extruded through a $50 \mathrm{~nm}$ membrane 21 times to obtain unilamellar vesicles. The size distribution was analyzed by dynamic light scattering. All solutions were degassed before beginning the experiments.

\section{Spectroscopy and Fluorescence Measurements.}

The pHLIP-PNA constructs were incubated overnight with POPC vesicles (1.5 mM lipid concentration) in PBS, $\mathrm{pH} 8$. To initiate transmembrane insertion, the $\mathrm{pH}$ of the mixture was decreased to $\mathrm{pH} 4$ by the addition of $1 \mathrm{M} \mathrm{HCl}$. Both before and after $\mathrm{HCl}$ addition, $\mathrm{CD}$ spectra were collected using a Chirascan ${ }^{\mathrm{TM}}$ CD Spectrometer from Applied Photophysics. Fluorescence spectra were recorded using a PTI QuantaMaster Spectrofluorometer with the 
spectral widths of the excitation and emission slits set at $2 \mathrm{~nm}$ and $1 \mathrm{~nm}$, respectively. For tryptophan fluorescence, an excitation wavelength of $280 \mathrm{~nm}$ was used. The concentrations of the pHLIP constructs were kept at $5 \mu \mathrm{M}$ for all tryptophan fluorescence and CD measurements. CD measurements were also performed for the PNAs alone (without pHLIP) incubated with POPC vesicles at $\mathrm{pH} 8$ and $\mathrm{pH} 4$, and fluorescence measurements in the tryptophan emission range upon excitation at $280 \mathrm{~nm}$ were performed for PNAs alone in $\mathrm{pH}$ 7.4 PBS. As expected, the PNAs exhibited no significant CD signal or fluorescence signal within the tryptophan emission range (SI Figure 2).

For TAMRA quenching assays, QSY-9 quencher with succinimidyl ester was purchased from ThermoFisher Scientific. The succinimidyl ester was removed by hydrolysis from incubation of the QSY-9 quencher in PBS at room temperature for 3 days. For all assay measurements, $2 \mu \mathrm{M}$ of pHLIP-PNA construct was used. 2 hours after the initiation of transmembrane insertion by $\mathrm{pH}$ reduction, $8 \mu \mathrm{M}$ QSY-9 quencher was added. TAMRA fluorescence before and after quenching was investigated by excitation at $557 \mathrm{~nm}$.

Fluorescence measurements were performed at 20 seconds, 1 minute, and 5 minutes after the addition of quencher. No change in fluorescence over time was observed. When the same concentration of quencher was added to the pHLIP-PNA constructs alone (without POPC vesicles), the fluorescence signal was instantaneously and completely quenched (SI Figure 2c). Together, these results suggested that there was no significant leakage of quencher through the POPC membrane over the given timescale. Therefore, the first fluorescence measurement at 20 seconds was used.

\section{Cell Culture.}

Human lung carcinoma (A549) cells were obtained from the American Type Culture Collection (ATCC). C57BL/6-syngeneic mouse melanoma (YUMM1.7) cells were previously derived in the lab of Marcus Bosenberg. ${ }^{32}$ For growth and maintenance, A549 and YUMM1.7 cells were cultured in Dulbecco's Modified Eagle Medium (DMEM) and DMEM/Ham's Nutrient Mixture F-12 (DMEM/F-12), respectively, supplemented with 10\% fetal bovine serum (Gibco), $1 \%$ penicillin, and $1 \%$ streptomycin in a $37^{\circ} \mathrm{C}, 5 \% \mathrm{CO}_{2}$ humidified incubator. All cells were cultured for less than 20 passages before use. After use for the experiments performed in this study, the cell lines were reauthenticated by the Yale DNA Analysis Core Facility (New Haven, CT, USA) using short tandem repeat analysis. During maintenance, cells were periodically verified to be mycoplasma negative using the MycoAlert Mycoplasma Detection kit (Lonza).

\section{Confocal Microscopy and Flow Cytometry.}

For $\mathrm{pH}$-controlled cell treatment assays in vitro, it was found that $\mathrm{pH}$ tends to drift in media relying upon a bicarbonate buffering system. Hence, all cell treatments were performed in L-15 Leibovitz media (Hyclone), which is primarily buffered by phosphate instead of bicarbonate. Immediately prior to treatment, A549 cells plated on a collagen-coated plate were washed once with $\mathrm{pH} 7.4$ or $\mathrm{pH} 6.2 \mathrm{~L}-15$ Leibovitz. Afterward, the media was aspirated, and fresh $\mathrm{pH} 7.4$ or $\mathrm{pH} 6.2 \mathrm{~L}-15$ Leibovitz containing the appropriate treatment (pHLIP-PNA, PNA alone, or pHLIP-TAMRA) at a $500 \mathrm{nM}$ concentration was added. The cells were subsequently incubated for 2 hours at $37^{\circ} \mathrm{C}$ in a humidified incubator without 
supplemental $\mathrm{CO}_{2}$. Following treatment, to prepare cells for confocal microscopy, the cells were washed twice with DPBS and then fixed in $4 \%$ paraformaldehyde. Fluorescence imaging was performed using a Zeiss LSM 510 confocal microscope equipped with a 561 $\mathrm{nm}$ excitation laser. For flow cytometry, the cells were washed twice with DPBS, trypsinized, and suspended in L-15 Leibovitz supplemented with 10\% FBS. They were then centrifuged and resuspended in 4\% paraformaldehyde in PBS for fixation. Flow cytometry was performed using an LSR II flow cytometer (BD Biosciences) equipped with a $532 \mathrm{~nm}$ excitation laser.

\section{Mouse Experiments.}

All animals used in this study were housed in clean facilities maintained by the Yale Animal Resource Center. All protocols were approved in advance by the Yale University Institutional Animal Care and Use Committee. 6-week old C57BL/6 mice were purchased from Jackson Laboratories (Bar Harbor, ME, USA) and allowed to acclimatize to the new facility for 2 weeks. Prior to the induction of tumors, C57BL/6-syngeneic YUMM1.7 cells were grown to $\sim 70 \%$ confluence. Afterward, the cells were suspended by trypsinization followed by the addition of normal growth media to neutralize the trypsin. The cells were then centrifuged and resuspended twice in DPBS to a concentration of $2.5 \times 10^{6}$ cells $/ \mathrm{mL}$. For each mouse, tumors were seeded by injecting $100 \mu \mathrm{L}$ of the resulting cell suspension subcutaneously into the left flank using a $27 \mathrm{G}$ needle.

After 3-4 weeks, the resulting tumors were $\sim 0.5 \mathrm{~cm}^{3}$ in size. At this point, the mice were intravenously injected via the retro-orbital sinus with $0.2 \mu$ moles $/ \mathrm{kg}$ ( $\sim 5$ nmoles $)$ of pHLIPPNA construct, PNA alone, or vehicle. After 24 hours, the mice were sacrificed, and their tumors and major organs were excised for imaging. Fluorescence imaging of the tumors and major organs was conducted using a CRi Maestro 2 Multispectral Imaging system (Cambridge Research Instrumentation, Woburn, MA). The images were spectrally unmixed and analyzed, and tumor fluorescence intensities were measured using the accompanying CRi Maestro software.

\section{Results and Discussion}

\section{Design of PNA Cargo Molecules.}

We designed PNAs of length 12 to 25 nucleobases and linked on either end to cysteine or the fluorophore TAMRA, respectively, via a short PEG linker (-ooo-). The cysteine allowed the PNA to be conjugated to the C-terminal cysteine of pHLIP via a disulfide bond, which is cleaved after entering the cytosol. To keep the PNAs similar for comparison, all PNAs were designed with similar purine content (37.5-50\%) and contained sequences that included the full sequence of any shorter PNAs used in the study. The resulting PNA cargoes had molecular weights ranging from $\sim 4-8 \mathrm{kDa}$. As cargo hydrophilicity directly affects pHLIP insertion, ${ }^{26}$ we measured the octanol-water distribution coefficient $(\operatorname{LogD})$ of each PNA at the $\mathrm{pH}$ values used in our experiments (Table 2). The PNAs were all found to be moderately hydrophilic and had approximately equal $\log \mathrm{D}$ values at each respective $\mathrm{pH}$. Of note, the LogD values were slightly higher at lower $\mathrm{pH}$, suggesting the presence of alternate, more hydrophobic protonation states. The result was likely increased membrane permeability. 
Hence, in the following experiments, protonation of the PNA cargo may also have been contributing to pHLIP transmembrane insertion. However, the impact is expected to have been modest, since the $\log \mathrm{D}$ for each PNA was only 0.3 to 0.4 units higher at $\mathrm{pH} 4.0$ compared to $\mathrm{pH} 7.4$.

\section{Interaction of pHLIP-PNA Constructs with Lipid Bilayers.}

We first examined whether each of the pHLIP-PNA constructs was capable of undergoing pH-dependent insertion into lipid bilayers. For this, we incubated each pHLIP-PNA construct with vesicles made from 1-palmitoyl-2-oleoyl-sn-glycero-3-phosphocholine (POPC). The mixtures were incubated overnight at $\mathrm{pH} 8$ to allow for State 2 binding of pHLIP to the lipid membranes. Afterwards, the $\mathrm{pH}$ of each mixture was decreased to $\mathrm{pH} 4$ to initiate pHLIP transmembrane insertion (State 3). It is well established that, during insertion, pHLIP shifts from an unstructured form in State 2 to an alpha helix in State 3, resulting in a significant change in circular dichroism to a spectrum characteristic of an alpha helix. ${ }^{5,33-40}$ Similarly, the fluorescence emission spectra of the two tryptophan residues in pHLIP's transmembrane region are blueshifted and undergo an increase in intensity upon transitioning from an aqueous environment to the lipid bilayer environment. ${ }^{5,33-40}$ Hence, transmembrane insertion was monitored by changes in both circular dichroism (CD) and tryptophan fluorescence (Figure 2).

The resulting data suggest that all four pHLIP-PNA constructs are capable of undergoing transmembrane insertion. Of note, the change in pHLIP's conformation to an alpha helix occurs prior to insertion, while pHLIP is still located on the outer leaflet of the membrane, ${ }^{33}$ and we therefore expected that the PNA cargoes would have little or no interference with this conformational step in the process. Our results are consistent with this hypothesis, as each of the pHLIP-PNA constructs exhibited changes in CD consistent with the formation of an alpha helix by the time they were first measured ( 2 minutes) (Figure 2a-d). However, the kinetics of transmembrane insertion, as determined by changes in tryptophan fluorescence, were significantly slowed by the presence of a PNA cargo. The characteristic time for insertion of pHLIP with no cargo is $\sim 40$ seconds. ${ }^{33,36,39}$ With PNAs, the time was increased to over an hour (Figure 2i). This was expected, given that prior studies with pHLIP linked to biotin, a very small cargo (molecular weight $=244.3 \mathrm{Da}$ ), showed an increase of over 10-fold in the characteristic time for insertion compared to pHLIP with no cargo. ${ }^{36}$ Likewise, the kinetics of insertion were significantly slower for larger PNA cargoes than for smaller cargoes. For the 25-mer PNA construct, tryptophan fluorescence intensity at 1 hour was approximately half that of the 12-mer PNA construct (Figure 2i). These results suggest that there is a practical limit in cargo size when it comes to pHLIP insertion, beyond which the kinetics are too slow for effective delivery to tumor cells.

Next, we compared the actual amounts of PNA that were inserted into vesicles by pHLIP at the 2-hour time point. To do this, we utilized a soluble, membrane-impermeable quencher, QSY-9, which effectively quenches TAMRA fluorescence. Addition of QSY-9 to each mixture allowed us to distinguish between uninserted and inserted TAMRA-labeled PNA, since the PNA that was inserted into vesicles by pHLIP would be protected from quenching of TAMRA fluorescence, while uninserted PNA would not. As expected, the degree of 
protection from quenching inversely correlated with PNA size (Figure 3). Approximately twice as much 12-mer PNA was inserted compared to 25-mer PNA.

\section{Delivery of PNA Cargoes to Cultured Cells.}

POPC vesicles are a well-established model for investigating lipid membrane interactions. Nevertheless, mammalian cell membranes are considerably different, with a composition that includes cholesterol, several different classes of lipids, and $~ 50 \%$ protein by weight or $>20 \%$ protein by area. ${ }^{41,42}$ Furthermore, it has been shown that both the State 2 binding and State 3 insertion of pHLIP into lipid vesicles can be significantly influenced by the presence of cholesterol or other lipids besides POPC. ${ }^{34,43,44}$ Thus, it is important to assess whether the findings in vesicles correspond to related events in cells. Therefore, we treated A549 cells with each of the pHLIP-PNA constructs at either $\mathrm{pH}$ 6.2, to simulate the acidic tumor microenvironment, or $\mathrm{pH} 7.4$, to simulate the interstitial fluid of normal tissue. Upon analyzing PNA delivery into the cells via the fluorescence of the TAMRA label according to both flow cytometry and confocal microscopy, we saw significantly increased PNA delivery at $\mathrm{pH} 6.2$ compared to $\mathrm{pH} 7.4$ for all but the 25-mer pHLIP-PNA (Figure 4). As expected, the PNAs alone were unable to enter cells at either $\mathrm{pH}$ (Figure 4c). In addition, comparison of cells treated with pHLIP-PNA to cells treated with pHLIP conjugated via a non-cleavable thioether bond to TAMRA (no PNA) provided evidence that disulfide bond reduction and consequent release of the PNA were occurring within cells upon transmembrane insertion. While cells treated with the non-cleavable pHLIP-TAMRA exhibited punctate fluorescence indicative of endocytic uptake, consistent with cell membrane localization (Figure 4d), the fluorescence from pHLIP-PNA(TAMRA) delivery was much more diffuse (Figure 4a), suggesting that release of the PNAs had occurred, allowing them to diffuse throughout the cell cytosol.

As with the kinetics of insertion into POPC vesicles, the ratio of PNA delivery at $\mathrm{pH} 6.2$ vs. delivery at $\mathrm{pH} 7.4$ was inversely correlated with PNA size (Figure 4e-f). While the 12-mer and 16-mer pHLIP-PNA treatments both yielded high $\mathrm{pH}$ 6.2-to-7.4 delivery ratios, this was reduced for the 20-mer pHLIP-PNA, and there was no apparent $\mathrm{pH}$-dependence for delivery of the 25-mer pHLIP-PNA. This observation strongly suggests that there is an upper limit in terms of the size of the cargo that pHLIP can deliver into cells in a pH-dependent fashion on the timescale of 2 hours, a practical limit for many applications. For moderately hydrophilic cargoes with similar LogDs to our PNAs, this limit appears to be around $7 \mathrm{kDa}$.

Interestingly, in addition to diffuse PNA fluorescence throughout the cell cytosol, we observed punctate TAMRA fluorescence for the 25-mer pHLIP-PNA and, to a lesser extent, for the 20-mer pHLIP-PNA (SI Figure 3). These puncta are again indicative of endocytosis. Their presence in cells treated with the longer length pHLIP-PNAs is consistent with slower kinetics of insertion. The longer it takes for pHLIP to undergo transmembrane insertion, the higher the chance that the pHLIP-PNA will be endocytosed before insertion occurs. Hence, it is expected that there would be more pHLIP-PNA in endosomes for the 20-mer, and even more for the 25-mer, than for the 12-mer and 16-mer pHLIP-PNAs. Given the presence of pHLIP-PNA in endosomes, the possibility of pHLIP acting as an endosomal escape device arises. If the rate of insertion is slow enough, such that a significant amount of the pHLIP- 
cargo is endocytosed before insertion occurs, then pHLIP transmembrane insertion through the endosomal membrane and into the cytosol may serve as an important pathway for drug delivery.

\section{Targeting and Delivery of PNA Cargoes to Tumors.}

We next investigated the influence of cargo size on pHLIP's ability to target tumors while avoiding normal tissue. In numerous previous studies, pHLIP has been shown to target tumors when linked to small fluorophores or imaging agents, but the influence of larger cargoes on tumor targeting remains unknown. Coupling a molecule of a size comparable to or larger than pHLIP ( 4.2 kDa) may have a significant effect on pHLIP's pharmacokinetics and biodistribution. In addition, although pHLIP-mediated tumor targeting is believed to be the result of $\mathrm{pH}$-dependent delivery, there are a variety of other factors which may contribute, such as the relative leakiness of blood vessels and poor lymphatic drainage within tumors, known as the enhanced-permeability-and-retention (EPR) effect. The EPR effect increases the tumor residence time of nanoparticles and many larger or protein-bound drugs, thereby increasing their tumor specificity. ${ }^{45-47}$ It is likely that pHLIP is influenced by the EPR effect as well, particularly when carrying larger cargoes. Hence, it is not at all obvious that pHLIP's ability to translocate molecules across cell membranes in a pHdependent manner will correspond with its ability to target tumors. While the delivery of PNAs to tumors by pHLIP was suggested in prior studies by the presence of a therapeutic effect, tumor targeting was never actually demonstrated. ${ }^{22,23}$ The possibility remains that, in these studies, the pHLIP-PNAs did not actually target tumors, but still accumulated to a sufficient degree within tumors to produce a therapeutic effect. We therefore investigated tumor targeting directly with our TAMRA-labeled pHLIP-PNA constructs.

Mice bearing melanoma tumors were intravenously injected with each pHLIP-PNA construct or the 16-mer PNA alone. 24 hours later, the mice were sacrificed, their tumors and major organs were excised, and the biodistribution of the PNAs was analyzed by fluorescence imaging (Figure 5). As can be seen, all the pHLIP-PNAs demonstrated excellent tumor targeting, as evidenced by the contrast in PNA signal between tumor and normal tissue. However, tumor delivery (indicated by the magnitude of the tumor signal) was significantly greater for the 12-mer and 16-mer pHLIP-PNAs than it was for the longer length pHLIP-PNAs. Delivery was approximately equal for the 12-mer and 16-mer pHLIPPNAs, but almost 3-fold lower for the 20-mer pHLIP-PNA, and even lower for the 25-mer pHLIP-PNA. Thus, there appears to be a threshold of cargo size between that of the 16-mer and 20-mer PNAs, over which pHLIP-mediated delivery drops significantly. This size corresponds to a cargo of approximately $6 \mathrm{kDa}$. Nevertheless, delivery of cargoes larger than this, although suboptimal, may still be sufficient to produce a therapeutic effect, as evidenced previously with a microRNA-inhibiting $23-\operatorname{mer}(\sim 7 \mathrm{kDa})$ PNA. $^{22}$

Of note, the 25-mer pHLIP-PNA still exhibited significant tumor targeting, despite its failure to undergo pH-mediated delivery into cultured cells. This observation might be explained in two ways. Firstly, the mouse experiment occurred over a longer time scale (24 hours) compared to our cell culture experiments ( 2 hours). Hence, there was much more time available for pHLIP-mediated translocation to occur. As our POPC vesicle experiments 
demonstrated, the kinetics of pHLIP insertion are dramatically slower for larger cargoes. Nevertheless, over the long time scales of in vivo experiments, tumor targeting can still occur, but overall intracellular delivery may still be reduced considerably.

Secondly, there may be $\mathrm{pH}$-independent processes that contribute to pHLIP's tumor targeting function, such as the aforementioned EPR effect. The EPR effect is expected to be significant for larger pHLIP constructs and pHLIP constructs bound to serum proteins. The fact that the 16-mer PNA alone also exhibited a small degree of tumor targeting further suggests that the EPR effect contributes. Indeed, it is possible that the EPR effect works synergistically with $\mathrm{pH}$-dependent insertion. One can posit that the EPR effect would increase the residence time of pHLIP within tumors, thereby increasing the likelihood of pHLIP undergoing $\mathrm{pH}$-dependent insertion. This synergy would be particularly prominent for more slowly inserting pHLIP constructs, such as those with large cargoes, since, if not for the EPR effect, these constructs would likely have insufficient time to undergo $\mathrm{pH}$ dependent insertion before being washed away from the tumor. In contrast, there would be nothing preventing the constructs from washing away in normal tissue. The result would be a significant increase in the specificity of tumor targeting. Interestingly, the tumor specificity we observed for our pHLIP-PNA constructs, particularly the 12-mer and 16-mer constructs, appears to be significantly better than what is typically seen for pHLIP when no cargo is present on the inserting end (see references ${ }^{9-14}$ ). Therefore, under certain conditions, large cargoes might actually increase the specificity of tumor targeting.

Also of interest, we did not see significant targeting of the kidneys with our pHLIP-PNA constructs, even though urine is typically acidic. This is in contrast to what is typically seen in pHLIP studies, in which pHLIP may accumulate in the kidneys to a degree comparable to its accumulation in tumors. ${ }^{9-14}$ A likely explanation for this is that the larger size of the constructs reduced their permeation through the glomerular filtration barrier. However, it is also possible that the slower kinetics of insertion reduced the likelihood of pHLIP being inserted into the kidneys before being washed away and excreted in the urine. Further study of exactly how insertion kinetics and the EPR effect influence pHLIP's biodistribution and tumor targeting could prove valuable.

\section{Conclusions}

In sum, using a set of moderately polar cargoes, we have investigated the influence of molecular size on $\mathrm{pH}$-mediated delivery and tumor targeting by pHLIP. We find that cargo size largely affects the kinetics and efficiency of $\mathrm{pH}$-dependent insertion across lipid membranes. For moderately polar cargoes such as PNAs, pHLIP is able to effectively translocate $\sim 7 \mathrm{kDa}$ across cell membranes. Of note, this was previously observed for pHLIP linked to a 23-mer (7.04 kDa) PNA designed to inhibit an oncogenic miRNA, which substantially reduced the tumor burdens of mice exhibiting lymphoma addicted to the miRNA.22 Furthermore, pHLIP seems to retain its tumor targeting capabilities when linked to cargoes of this size, and we speculate that, in certain circumstances, the specificity of tumor targeting may even be enhanced compared to pHLIP with no linked cargo. However, the actual amount of a molecular cargo that pHLIP delivers to tumors seems to decrease 
considerably for moderately polar cargoes of greater than $\sim 6 \mathrm{kDa}$, presumably due to the slower kinetics of insertion.

Of note, these values for cargo size serve only as rough guidelines, as our study was limited to PNA cargoes with specific properties. Cargo polarity is also known to have a significant effect on pHLIP delivery. ${ }^{26}$ It is possible that pHLIP can deliver larger molecules if they are more hydrophobic, given that lipid membrane permeability correlates with hydrophobicity. ${ }^{48}$ In addition, $\mathrm{pH}$ may affect a cargo's hydrophobicity. As we noted earlier, our PNAs exhibited slightly more positive $\operatorname{LogD}$ values at lower $\mathrm{pH}$, indicating slightly higher hydrophobicities. Hence, care should be taken when applying these results to other molecules, even other PNAs. For instance, the presence of TAMRA on the PNAs used in this study may have influenced their hydrophobicity and hence delivery by pHLIP. Previously, it was found that the inclusion of TAMRA significantly enhanced pHLIP-mediated delivery of phalloidin, presumably by making the cargo more hydrophobic. ${ }^{16}$ It is possible that a similar effect occurs with the PNAs.

Furthermore, pHLIP-mediated delivery may be influenced by additional charge and conformation factors that were not considered in this study. For instance, PNAs are typically unstructured in solution, but can be modified to possess a helical conformation by adding a chirality-inducing group to the $\gamma$-carbon of each respective PNA monomer in the peptide backbone of the PNA. ${ }^{49}$ It is unclear what effect this change in conformation would have on pHLIP-mediated delivery. In addition, molecules can adapt conformations that mask charged groups within their interiors, thereby increasing their lipid membrane permeability. ${ }^{1}$ Finally, the composition of the cell membrane, along with membrane fluidity, can vary substantially. ${ }^{50}$ Both lipid composition and membrane physical properties have previously been noted to have significant effects on pHLIP transmembrane insertion. ${ }^{34,43,44}$

For all these reasons, the limiting values for the molecular weights of pHLIP cargoes may vary substantially, and the values determined in this study can only serve as rough guidelines. Regardless, our results demonstrate that the range of sizes for viable pharmaceuticals can be vastly expanded by coupling such molecules to pHLIP. Together with targeting, the inclusion of more polar and larger agents could usefully expand the range of effective therapies in cancer and other acidic pathologies.

\section{Supplementary Material}

Refer to Web version on PubMed Central for supplementary material.

\section{Acknowledgments}

Work in the lab of D.M.E. is supported by NIH grant R01-GM073857. A.A.S. was additionally supported by a NIH National Research Service Award (F30-CA196020) and Yale University's NIH Medical Scientist Training Program grant (T32-GM007205). P.M.G. is supported by NIH grants R01-ES005775 and R35-CA197574. D.D. and M.C.B. were supported by R01-CA037157. R.B. is supported by University of Connecticut startup funds, the UConn START PPOC award, and a St. Baldrick's Foundation childhood cancer research grant. 


\section{References}

1. Yang NJ; Hinner MJ Getting across the cell membrane: an overview for small molecules, peptides, and proteins. Methods Mol Biol 2015, 1266, 29-53. [PubMed: 25560066]

2. Lipinski CA; Lombardo F; Dominy BW; Feeney PJ Experimental and computational approaches to estimate solubility and permeability in drug discovery and development settings. Adv Drug Deliv Rev 2001, 46, (1-3), 3-26. [PubMed: 11259830]

3. Hajnal K; Gabriel H; Aura R; Erzsébet V; Blanka SS Prodrug Strategy in Drug Development. Acta Medica Marisiensis 2016, 62, (3), 356-362.

4. Barua S; Mitragotri S Challenges associated with Penetration of Nanoparticles across Cell and Tissue Barriers: A Review of Current Status and Future Prospects. Nano Today 2014, 9, (2), 223 243. [PubMed: 25132862]

5. Hunt JF; Rath P; Rothschild KJ; Engelman DM Spontaneous, pH-dependent membrane insertion of a transbilayer alpha-helix. Biochemistry 1997, 36, (49), 15177-92. [PubMed: 9398245]

6. Deacon JC; Engelman DM; Barrera FN Targeting acidity in diseased tissues: mechanism and applications of the membrane-inserting peptide, pHLIP. Arch Biochem Biophys 2015, 565, 40-8. [PubMed: 25444855]

7. Liberti MV; Locasale JW The Warburg Effect: How Does it Benefit Cancer Cells? Trends Biochem Sci 2016, 41, (3), 211-218. [PubMed: 26778478]

8. Wei D; Engelman DM; Reshetnyak YK; Andreev OA Mapping pH at Cancer Cell Surfaces. Mol Imaging Biol 2019.

9. Adochite RC; Moshnikova A; Golijanin J; Andreev OA; Katenka NV; Reshetnyak YK Comparative Study of Tumor Targeting and Biodistribution of $\mathrm{pH}$ (Low) Insertion Peptides (pHLIP((R)) Peptides) Conjugated with Different Fluorescent Dyes. Mol Imaging Biol 2016, 18, (5), 686-96. [PubMed: 27074841]

10. Daumar P; Wanger-Baumann CA; Pillarsetty N; Fabrizio L; Carlin SD; Andreev OA; Reshetnyak YK; Lewis JS Efficient (18)F-labeling of large 37-amino-acid pHLIP peptide analogues and their biological evaluation. Bioconjug Chem 2012, 23, (8), 1557-66. [PubMed: 22784215]

11. Demoin DW; Wyatt LC; Edwards KJ; Abdel-Atti D; Sarparanta M; Pourat J; Longo VA; Carlin SD; Engelman DM; Andreev OA; Reshetnyak YK; Viola-Villegas N; Lewis JS PET Imaging of Extracellular $\mathrm{pH}$ in Tumors with (64)Cu- and (18)F-Labeled pHLIP Peptides: A Structure-Activity Optimization Study. Bioconjug Chem 2016, 27, (9), 2014-23. [PubMed: 27396694]

12. Macholl S; Morrison MS; Iveson P; Arbo BE; Andreev OA; Reshetnyak YK; Engelman DM; Johannesen E In vivo pH imaging with (99m)Tc-pHLIP. Mol Imaging Biol 2012, 14, (6), 725-34. [PubMed: 22371188]

13. Reshetnyak YK; Yao L; Zheng S; Kuznetsov S; Engelman DM; Andreev OA Measuring tumor aggressiveness and targeting metastatic lesions with fluorescent pHLIP. Mol Imaging Biol 2011, 13, (6), 1146-56. [PubMed: 21181501]

14. Vavere AL; Biddlecombe GB; Spees WM; Garbow JR; Wijesinghe D; Andreev OA; Engelman DM; Reshetnyak YK; Lewis JS A novel technology for the imaging of acidic prostate tumors by positron emission tomography. Cancer research 2009, 69, (10), 4510-6. [PubMed: 19417132]

15. Karabadzhak AG; An M; Yao L; Langenbacher R; Moshnikova A; Adochite RC; Andreev OA; Reshetnyak YK; Engelman DM pHLIP-FIRE, a cell insertion-triggered fluorescent probe for imaging tumors demonstrates targeted cargo delivery in vivo. ACS Chem Biol 2014, 9, (11), 2545-53. [PubMed: 25184440]

16. An M; Wijesinghe D; Andreev OA; Reshetnyak YK; Engelman DM pH-(low)-insertion-peptide (pHLIP) translocation of membrane impermeable phalloidin toxin inhibits cancer cell proliferation. Proceedings of the National Academy of Sciences of the United States of America 2010, 107, (47), 20246-50. [PubMed: 21048084]

17. Moshnikova A; Moshnikova V; Andreev OA; Reshetnyak YK Antiproliferative effect of pHLIPamanitin. Biochemistry 2013, 52, (7), 1171-8. [PubMed: 23360641]

18. Onyango JO; Chung MS; Eng CH; Klees LM; Langenbacher R; Yao L; An M Noncanonical Amino Acids to Improve the $\mathrm{pH}$ Response of pHLIP Insertion at Tumor Acidity. Angewandte Chemie 2015. 
19. Burns KE; McCleerey TP; Thevenin D pH-Selective Cytotoxicity of pHLIP-Antimicrobial Peptide Conjugates. Sci Rep 2016, 6, 28465. [PubMed: 27334357]

20. Burns KE; Hensley H; Robinson MK; Thevenin D Therapeutic Efficacy of a Family of pHLIPMMAF Conjugates in Cancer Cells and Mouse Models. Mol Pharm 2017, 14, (2), 415-422. [PubMed: 28048942]

21. Burns KE; Robinson MK; Thevenin D Inhibition of cancer cell proliferation and breast tumor targeting of pHLIP-monomethyl auristatin E conjugates. Mol Pharm 2015, 12, (4), 1250-8. [PubMed: 25741818]

22. Cheng CJ; Bahal R; Babar IA; Pincus Z; Barrera F; Liu C; Svoronos A; Braddock DT; Glazer PM; Engelman DM; Saltzman WM; Slack FJ MicroRNA silencing for cancer therapy targeted to the tumour microenvironment. Nature 2015, 518, (7537), 107-10. [PubMed: 25409146]

23. Ozes AR; Wang Y; Zong X; Fang F; Pilrose J; Nephew KP Therapeutic targeting using tumor specific peptides inhibits long non-coding RNA HOTAIR activity in ovarian and breast cancer. Sci Rep 2017, 7, (1), 894. [PubMed: 28420874]

24. Reshetnyak YK; Andreev OA; Lehnert U; Engelman DM Translocation of molecules into cells by $\mathrm{pH}$-dependent insertion of a transmembrane helix. Proceedings of the National Academy of Sciences of the United States of America 2006, 103, (17), 6460-5. [PubMed: 16608910]

25. Reshetnyak YK; Andreev OA; Segala M; Markin VS; Engelman DM Energetics of peptide (pHLIP) binding to and folding across a lipid bilayer membrane. Proceedings of the National Academy of Sciences of the United States of America 2008, 105, (40), 15340-5. [PubMed: 18829441]

26. Thevenin D; An M; Engelman DM pHLIP-mediated translocation of membrane-impermeable molecules into cells. Chemistry \& biology 2009, 16, (7), 754-62. [PubMed: 19635412]

27. Nielsen PE; Egholm M; Berg RH; Buchardt O Sequence-selective recognition of DNA by strand displacement with a thymine-substituted polyamide. Science 1991, 254, (5037), 1497-500. [PubMed: 1962210]

28. Babar IA; Cheng CJ; Booth CJ; Liang X; Weidhaas JB; Saltzman WM; Slack FJ Nanoparticlebased therapy in an in vivo microRNA-155 (miR-155)-dependent mouse model of lymphoma. Proceedings of the National Academy of Sciences of the United States of America 2012, 109, (26), E1695-704. [PubMed: 22685206]

29. Dean DA Peptide nucleic acids: versatile tools for gene therapy strategies. Adv Drug Deliv Rev 2000, 44, (2-3), 81-95. [PubMed: 11072107]

30. Fabani MM; Abreu-Goodger C; Williams D; Lyons PA; Torres AG; Smith KG; Enright AJ; Gait MJ; Vigorito E Efficient inhibition of miR-155 function in vivo by peptide nucleic acids. Nucleic Acids Res 2010, 38, (13), 4466-75. [PubMed: 20223773]

31. Fabani MM; Gait MJ miR-122 targeting with LNA/2'-O-methyl oligonucleotide mixmers, peptide nucleic acids (PNA), and PNA-peptide conjugates. RNA 2008, 14, (2), 336-46. [PubMed: 18073344]

32. Meeth K; Wang JX; Micevic G; Damsky W; Bosenberg MW The YUMM lines: a series of congenic mouse melanoma cell lines with defined genetic alterations. Pigment cell \& melanoma research 2016, 29, (5), 590-7. [PubMed: 27287723]

33. Andreev OA; Karabadzhak AG; Weerakkody D; Andreev GO; Engelman DM; Reshetnyak YK pH (low) insertion peptide (pHLIP) inserts across a lipid bilayer as a helix and exits by a different path. Proceedings of the National Academy of Sciences of the United States of America 2010, 107, (9), 4081-6. [PubMed: 20160113]

34. Barrera FN; Fendos J; Engelman DM Membrane physical properties influence transmembrane helix formation. Proceedings of the National Academy of Sciences of the United States of America 2012, 109, (36), 14422-7. [PubMed: 22908237]

35. Barrera FN; Weerakkody D; Anderson M; Andreev OA; Reshetnyak YK; Engelman DM Roles of carboxyl groups in the transmembrane insertion of peptides. J Mol Biol 2011, 413, (2), 359-71. [PubMed: 21888917]

36. Karabadzhak AG; Weerakkody D; Wijesinghe D; Thakur MS; Engelman DM; Andreev OA; Markin VS; Reshetnyak YK Modulation of the pHLIP transmembrane helix insertion pathway. Biophys J 2012, 102, (8), 1846-55. [PubMed: 22768940] 
37. Musial-Siwek M; Karabadzhak A; Andreev OA; Reshetnyak YK; Engelman DM Tuning the insertion properties of pHLIP. Biochimica et biophysica acta 2010, 1798, (6), 1041-6. [PubMed: 19766589]

38. Scott HL; Nguyen VP; Alves DS; Davis FL; Booth KR; Bryner J; Barrera FN The negative charge of the membrane has opposite effects on the membrane entry and exit of $\mathrm{pH}$-low insertion peptide. Biochemistry 2015, 54, (9), 1709-12. [PubMed: 25692747]

39. Scott HL; Westerfield JM; Barrera FN Determination of the Membrane Translocation pK of the pH-Low Insertion Peptide. Biophys J 2017, 113, (4), 869-879. [PubMed: 28834723]

40. Shu NS; Chung MS; Yao L; An M; Qiang W Residue-specific structures and membrane locations of pH-low insertion peptide by solid-state nuclear magnetic resonance. Nat Commun 2015, 6, 7787. [PubMed: 26195283]

41. Dupuy AD; Engelman DM Protein area occupancy at the center of the red blood cell membrane. Proceedings of the National Academy of Sciences of the United States of America 2008, 105, (8), 2848-52. [PubMed: 18287056]

42. Spector AA; Yorek MA Membrane lipid composition and cellular function. J Lipid Res 1985, 26, (9), 1015-35. [PubMed: 3906008]

43. Kyrychenko A; Vasquez-Montes V; Ulmschneider MB; Ladokhin AS Lipid headgroups modulate membrane insertion of pHLIP peptide. Biophys J 2015, 108, (4), 791-794. [PubMed: 25692583]

44. Vasquez-Montes V; Gerhart J; King KE; Thevenin D; Ladokhin AS Comparison of lipid-dependent bilayer insertion of pHLIP and its P20G variant. Biochim Biophys Acta Biomembr 2018, 1860, (2), 534-543. [PubMed: 29138065]

45. Kobayashi H; Watanabe R; Choyke PL Improving conventional enhanced permeability and retention (EPR) effects; what is the appropriate target? Theranostics 2013, 4, (1), 81-9. [PubMed: 24396516]

46. Maeda H; Nakamura H; Fang J The EPR effect for macromolecular drug delivery to solid tumors: Improvement of tumor uptake, lowering of systemic toxicity, and distinct tumor imaging in vivo. Adv Drug Deliv Rev 2013, 65, (1), 71-9. [PubMed: 23088862]

47. Matsumura Y; Maeda H A new concept for macromolecular therapeutics in cancer chemotherapy: mechanism of tumoritropic accumulation of proteins and the antitumor agent smancs. Cancer research 1986, 46, (12 Pt 1), 6387-92. [PubMed: 2946403]

48. Walter A; Gutknecht J Permeability of small nonelectrolytes through lipid bilayer membranes. J Membr Biol 1986, 90, (3), 207-17. [PubMed: 3735402]

49. Dragulescu-Andrasi A; Rapireddy S; Frezza BM; Gayathri C; Gil RR; Ly DH A simple gammabackbone modification preorganizes peptide nucleic acid into a helical structure. J Am Chem Soc 2006, 128, (31), 10258-67. [PubMed: 16881656]

50. Deliconstantinos G Physiological aspects of membrane lipid fluidity in malignancy. Anticancer Res 1987, 7, (5B), 1011-21. [PubMed: 3324933] 


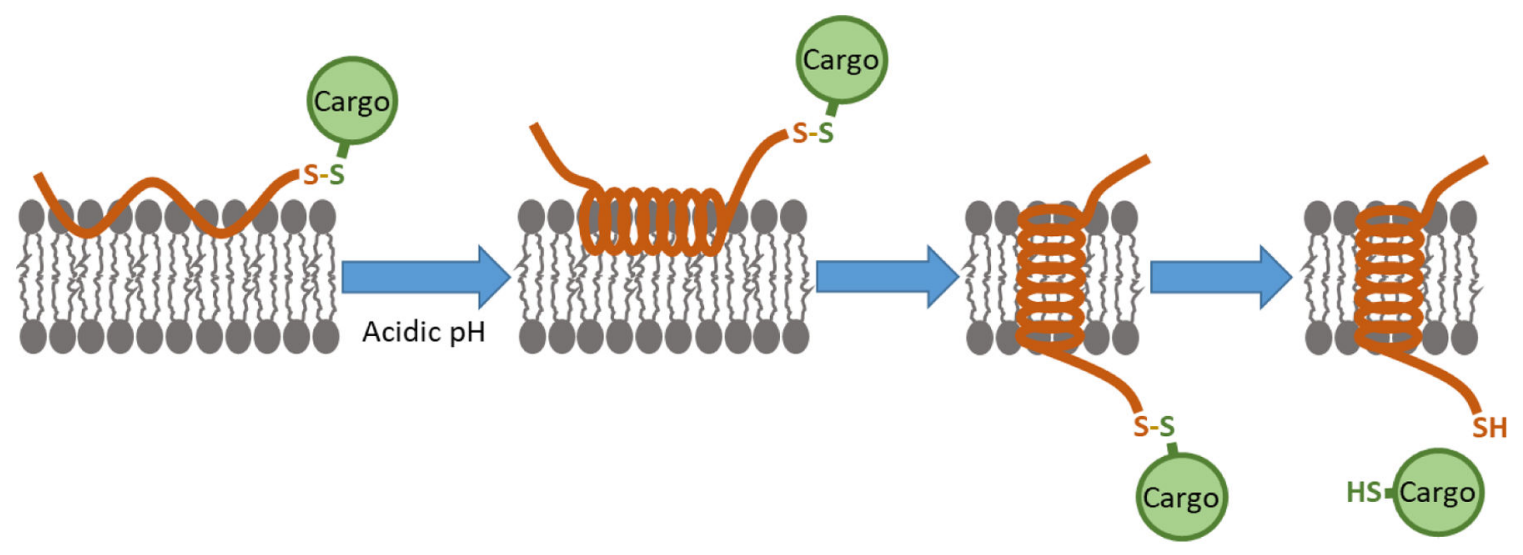

Figure 1: Diagram of pHLIP Transmembrane Insertion.

pHLIP can exist in three states: unstructured in solution (State 1) (not shown), docked at a cell membrane but not inserted (State 2) (left), and inserted across a cell membrane as a transmembrane alpha helix (State 3) (right). At normal physiologic pH, pHLIP exists in an equilibrium between States 1 and 2. At acidic pH, State 2 pHLIP adopts an alpha helical conformation and then transitions to State 3 via transmembrane insertion. A cargo molecule can be attached to the inserting end of pHLIP via a disulfide bond, which, after transmembrane insertion, is reduced in the cell cytoplasm, thereby releasing the cargo molecule. 

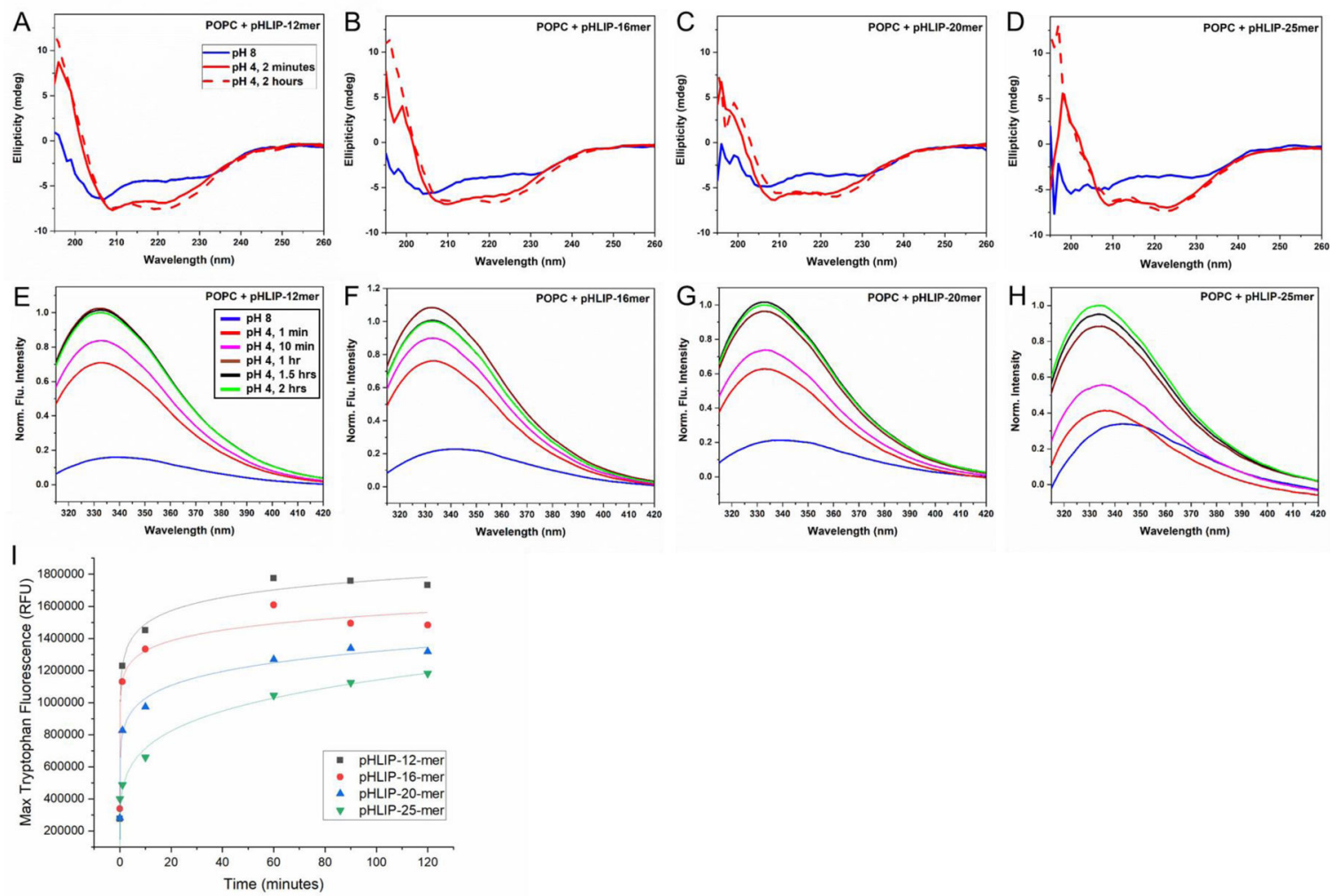

Figure 2: Interactions of pHLIP-PNA constructs with lipid bilayers.

CD spectroscopy (A-D) and tryptophan fluorescence measurements $(\mathrm{E}-\mathrm{H})$ at various time points before and after reducing $\mathrm{pH}$ from 8 to 4 suggest that pHLIP-PNA constructs incubated with POPC vesicles undergo $\mathrm{pH}$-triggered alpha-helix formation and transmembrane insertion, respectively. Maximum tryptophan fluorescence over time measurements (I) reveal differential kinetics of insertion for various pHLIP-PNA constructs. 

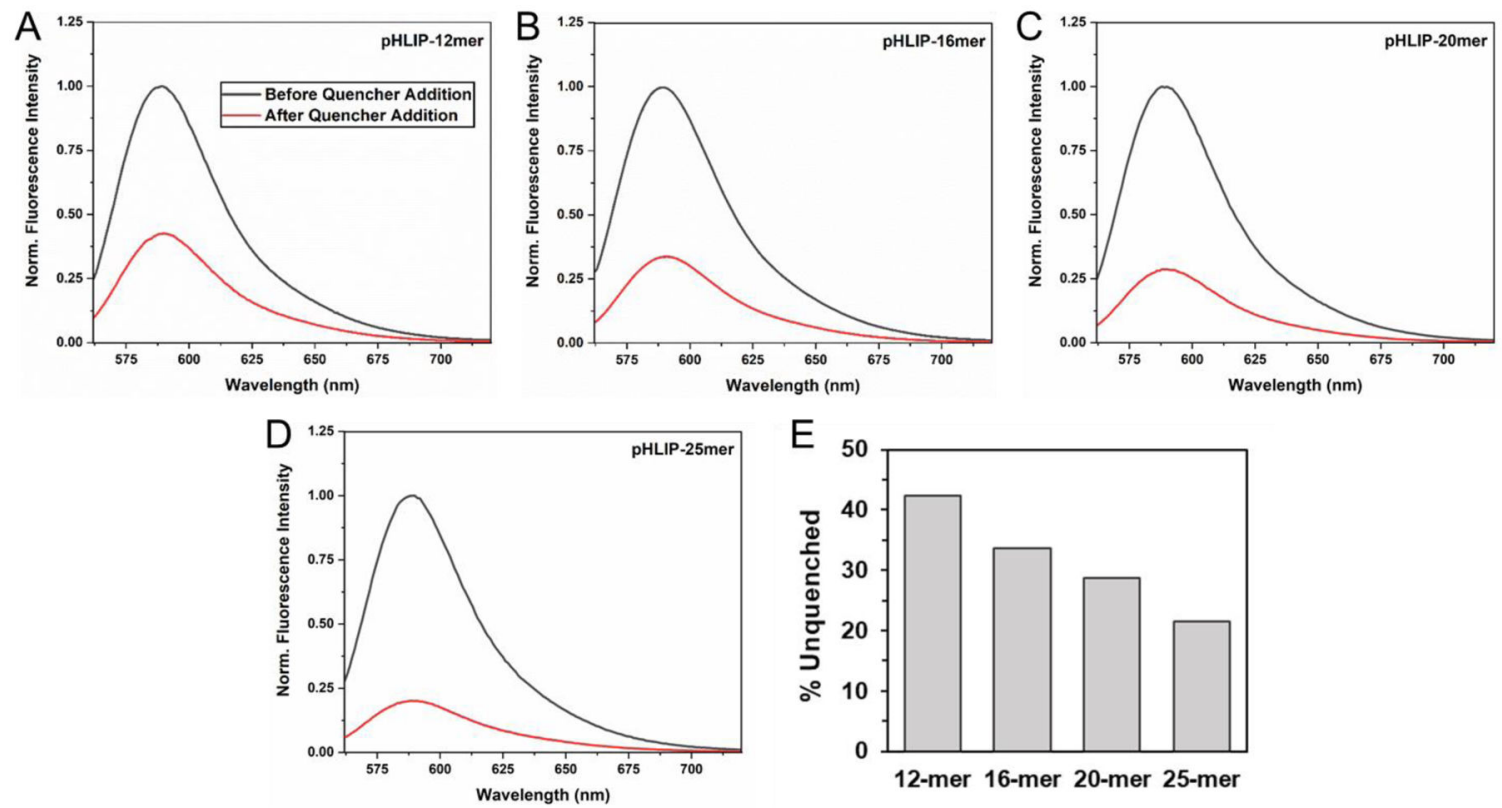

Figure 3: Relative amounts of PNA cargoes delivered across lipid bilayers by pHLIP after 2 hours.

pHLIP-PNA constructs incubated with POPC vesicles were allowed to undergo transmembrane insertion at $\mathrm{pH} 4$ for 2 hours prior to the addition of lipid membraneimpermeable QSY-9 quencher. The relative TAMRA fluorescence before (black) and after (red) quencher addition are shown (A-D). The \% unquenched for each construct was quantified by the relative area under the respective tryptophan fluorescence curve (E). 

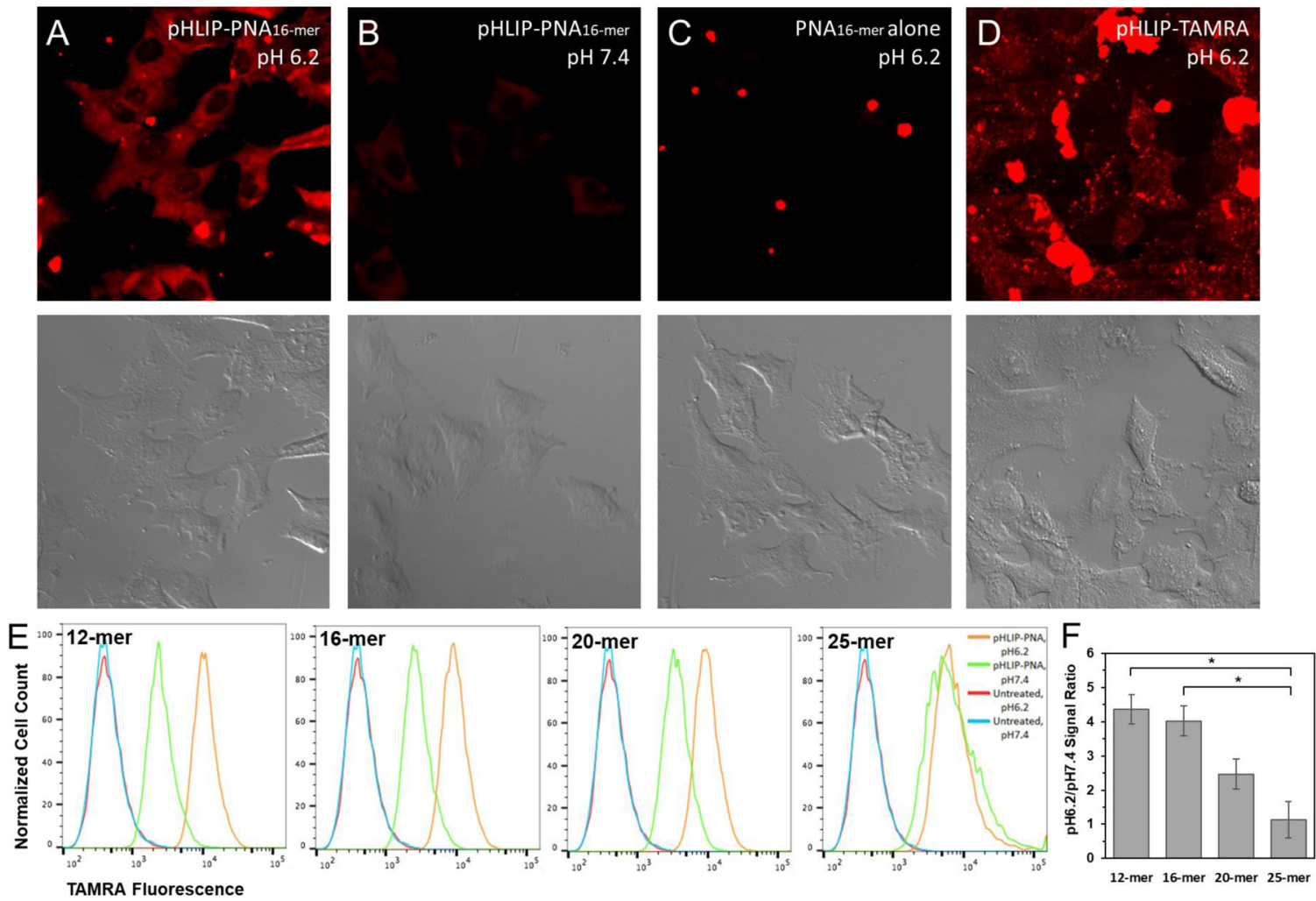

Figure 4: pHLIP delivers PNA cargoes to cultured cells in a pH-dependent fashion. A549 cells were incubated with pHLIP constructs or PNA alone at $\mathrm{pH} 6.2$ or $\mathrm{pH} 7.4$ for 2 hours. Confocal microscopy of TAMRA fluorescence (top) and accompanying DIC images (bottom) are shown for the 16-mer pHLIP-PNA construct at pH 6.2 (A) and $\mathrm{pH} 7.4$ (B), the 16-mer PNA alone at pH 6.2 (C), and pHLIP conjugated to TAMRA by a non-cleavable bond (D). Flow cytometry was used to quantify the relative amount of PNA delivered at $\mathrm{pH}$ 6.2 vs. $\mathrm{pH} 7.4$ for each pHLIP-PNA construct (E). The respective $\mathrm{pH}$ 6.2-to-pH 7.4 delivery ratios are compared in $(\mathrm{F})$. Data are shown as mean \pm S.E. $(\mathrm{n}=3) ;{ }^{*} \mathrm{P}<0.05$. 

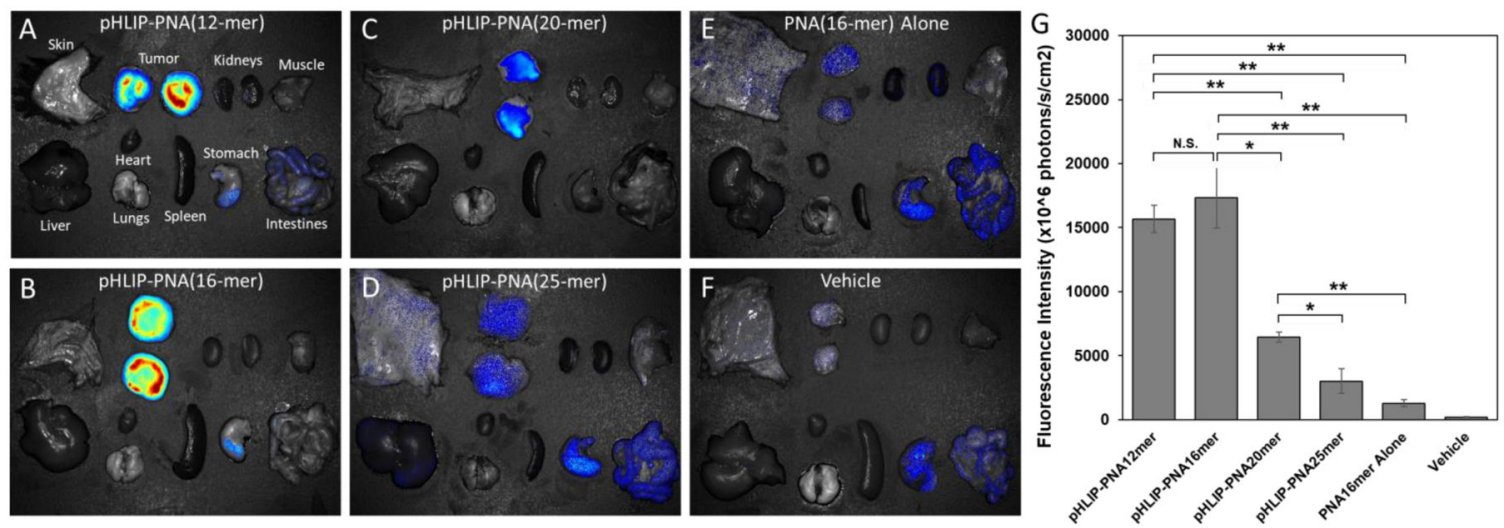

Figure 5: Targeted delivery of PNA cargoes to tumors by pHLIP.

pHLIP-PNA constructs were injected intravenously into melanoma tumor-bearing mice. 24 hours later, the mice were sacrificed and their tumors and major organs were excised and imaged for TAMRA fluorescence (A-F). Prior to imaging, the tumors were split in half to avoid obstruction of the fluorescence by the overlying skin, and the fluorescence intensities per unit area of the resulting two halves were averaged $(G)$. Data are shown as mean \pm S.E. $(\mathrm{n}=3) ; * \mathrm{P}<0.05 ; * * \mathrm{P}<0.01$. Of note, the signal in the stomach and intestines is present in the vehicle sample, indicating that it is tissue autofluorescence. 
Table 1:

Properties of cargoes that have previously been delivered by pHLIP

\begin{tabular}{|c|c|c|c|}
\hline Cargo & $\log P / \log D$ & $\begin{array}{l}\text { Molecular } \\
\text { Weight }\end{array}$ & Reference \\
\hline \multicolumn{4}{|l|}{ Cytotoxic Agents } \\
\hline Amanitin (SPDP linker) & -0.2 & 1007.1 & Moshnikova et al., 2013 (Ref 17) \\
\hline Amanitin (Lc-SMPT linker) & 0.2 & 1196.36 & Moshnikova et al., 2013 (Ref 17) \\
\hline Phalloidin, TAMRA (via separate linkers) & $-1.5,1.58^{*}$ & 1305.49 & An et al., 2010 (Ref 16$)$ \\
\hline Phalloidin-TRITC & -0.05 & 1305.57 & Reshetnyak et al., 2006 (Ref 24) \\
\hline Monomethyl Auristatin E & 2.2 & 692.96 & Burns et al., 2015 (Ref 21) \\
\hline Monomethyl Auristatin F & 0.7 & 806.07 & Burns et al., 2017 (Ref 20) \\
\hline \multicolumn{4}{|l|}{ Peptide Nucleic Acids } \\
\hline TAMRA-oo-CATAGTATAAGT-o-Cys & & 4055.08 & Reshetnyak et al., 2006 (Ref 24) \\
\hline ooo-TCTGTAACTC-ooo-Cys-Cy3 (Anti-HOTAIR PNA) & & 3635.24 & Özeş et al., 2017 (Ref 23) \\
\hline $\begin{array}{l}\text { TAMRA-ooo-ACCCCTATCACAATTA GCATTAA-ooo-Cys (Anti- } \\
\text { miR-155 PNA) }\end{array}$ & & 7036.94 & Cheng et al., 2015 (Ref 22) \\
\hline \multicolumn{4}{|l|}{ Fluorophores } \\
\hline Dansyl & $-1.43^{*}$ & 354.44 & Reshetnyak et al., 2006 (Ref 24) \\
\hline TAMRA & $1.58^{*}$ & 489.59 & Karabadzhak et al., 2014 (Ref 15) \\
\hline 2x TAMRA (via separate linkers) & $1.58^{*}, 1.58^{*}$ & 979.18 & Karabadzhak et al., 2014 (Ref 15) \\
\hline TAMRA, QSY-9 (via separate linkers) & $1.58^{*}, 0.98^{*}$ & 1367.63 & Karabadzhak et al., 2014 (Ref 15) \\
\hline Nitrobenzoxadiazole (NBD) & -0.83 & 293.3 & Thevenin et al., 2009 (Ref 26) \\
\hline \multicolumn{4}{|l|}{ Cyclic Peptides } \\
\hline NBD-cyclic(Ser) $)_{4}$ & -2.68 & 742.7 & Thevenin et al., 2009 (Ref 26) \\
\hline NBD-cyclic(Asp $)_{4}$ & -2.87 & 854.8 & Thevenin et al., 2009 (Ref 26) \\
\hline
\end{tabular}


Table 2.

Properties of PNA Cargo Molecules:

\begin{tabular}{|c|c|c|c|c|c|}
\hline PNA & Sequence & $\begin{array}{c}\text { Molecular } \\
\text { Weight }\end{array}$ & $\begin{array}{c}\text { LogD } \\
\mathbf{p H} \mathbf{7 . 4}\end{array}$ & $\begin{array}{c}\text { LogD } \\
\mathbf{p H} \mathbf{6 . 2}\end{array}$ & $\begin{array}{c}\text { LogD } \\
\mathbf{p H} \mathbf{4 . 0}\end{array}$ \\
\hline 12-mer & TAMRA-ooo-ACCATT GCCAAA-ooo-Cys & 4116.17 & -1.68 & -1.44 & -1.37 \\
\hline 16-mer & TAMRA-ooo-TTCTACCATTGCCAAA-ooo-Cys & 5166.18 & -1.78 & -1.59 & -1.43 \\
\hline 20-mer & TAMRA-ooo-TGAGTTCTACCATTGCCAAA-ooo-Cys & 6290.25 & -1.62 & -1.41 & -1.21 \\
\hline 25-mer & TAMRA-ooo-CGGT GT GAGTTCTACCATTGCCAAA-ooo-Cys & 7681.57 & -1.53 & -1.37 & -1.18 \\
\hline
\end{tabular}

